\title{
Uso de la evaluación en un contexto de múltiples agentes: el caso del plan de desarrollo rural del País Vasco en el marco de la Unión Europea
}

\author{
María Ángeles Díez \\ Universidad del País Vasco \\ mariaangeles.diez@ehu.es \\ Beatriz Izquierdo \\ Universidad de Burgos \\ bizquierdo@ubu.es \\ Eduardo Malagón \\ Universidad del País Vasco \\ eduardo.malagon@ehu.es
}

\begin{abstract}
Resumen
Este artículo analiza la utilización de los resultados y del proceso seguido en la evaluación de un programa de desarrollo rural basada en un enfoque participativo. Para ello, se utiliza la experiencia de la Evaluación Intermedia del Plan de Desarrollo Rural Sostenible del País Vasco 2000-2006. La premisa fundamental del equipo evaluador es que la participación incrementa la utilización de la evaluación, favoreciendo su uso en el diseño y en la toma de decisiones. El artículo se estructura en dos partes fundamentales: la primera de ellas describe la adecuación del modelo de evaluación participativa a los intereses y propósitos de nuestra evaluación. La segunda parte examina el impacto que este modelo de evaluación tuvo sobre el uso de sus hallazgos y recomendaciones. Esta valoración permitió extraer lecciones que pueden ayudar a reforzar el uso de la evaluación en contextos donde participan múltiples agentes, favoreciendo así el desarrollo de la gobernanza.
\end{abstract}

Palabras clave Uso de la evaluación, proceso de evaluación, cultura de evaluación, evaluación participativa, Política Europea de Desarrollo Rural, gobernanza.

\section{The Use of Evaluation in a Multi-Actor Context: The Case of the Rural Development Plan in The Basque Country (Within the European Framework)}

\begin{abstract}
This paper analyses the use of the evaluation results and the process carried out in the evaluation of a rural development evaluation programme focused in a participatory evaluation model. To do this, it is used the experience of the mid-term evaluation of the Sustainable Rural Development Plan 2000-2006 in the Basque Country. The core premise of the evaluation team was that stakeholder participation would enhance evaluation use, promoting its utilisation in the decision making process in future policies. The paper is structured into two main parts: The first one describes the adequacy of the participatory evaluation model to the interest and purposes of our evaluation. The second part examines the impact of this evaluation model on the evaluation utilisation. This assessment has given us the opportunity to draw lessons that can help to improve the utility of evaluation in multi - actor contexts, favouring thus the development of governance.
\end{abstract}

Key words

Use of evaluation, evaluation process, evaluation culture, participatory evaluation, European Rural Development Policy, Governance. 


\section{INTRODUCCIÓN}

Desde hace casi tres décadas, la utilidad de la evaluación ha sido objeto de un intenso debate dentro de la comunidad evaluadora, apareciendo diferentes visiones acerca de sus usos potenciales y reales. Una de las primeras cuestiones que surgen dentro de este debate es la idea de que el uso de los resultados y del propio proceso de la evaluación está intrínsecamente unido a los propósitos que guían cada evaluación. En definitiva, cada propuesta evaluadora, cuando define las funciones de la evaluación, va a determinar el modo en el que sus resultados van a ser utilizados (Alkin et al., 2006; Chelimsky, 1997; Chelimsky, 2006; Monnier, 1995; Patton, 2008; Weiss, 1998).

Además, en los últimos años, este creciente interés por la utilización de la evaluación ha puesto de relevancia la importancia que la tarea de difusión de las conclusiones y de los resultados, durante y después del proceso de evaluación, tiene a la hora de vincular evaluación y aprendizaje y contribuir así a que la evaluación genere el tipo de conocimiento usado por las personas que toman las decisiones políticas y/o son responsables del diseño y de la aplicación de las políticas (Torres et al., 2005; Alkin et al., 2006). En este contexto, una parte de la comunidad evaluadora ha ido progresivamente centrando su atención en la contribución de la evaluación al aprendizaje individual, interactivo y organizacional, promoviendo una perspectiva que provee de nuevos conceptos y ángulos de visión acerca del uso de la evaluación y enriquece el debate sobre la utilización (Cousins y Shulba, 2006; Torres et al., 2005; Patton, 1998; Patton, 2008).

Por tanto, la preocupación por la utilidad de la evaluación se materializa en el análisis y en el estudio de cuestiones que tienen que ver con los propósitos que deben guiar una determinada evaluación o, dicho de otra manera, con la función o funciones que la evaluación puede y debe desempeñar. Como algunas autoras destacan, los propósitos de la evaluación no suelen ser rígidos o fijados y los equipos de evaluación de los programas disponen de opciones (Weiss, 1998; Chelimsky, 2006). Los equipos de evaluación pueden hacer sugerencias acerca de las preguntas centrales de la evaluación y sus posibles usos y pueden negociar estos elementos evaluadores con las audiencias de la evaluación (stakeholders): con las personas o grupos implicados y afectados por el proceso de evaluación de forma directa o indirecta.

En efecto, los stakeholders ocupan un lugar central en el debate sobre la utilización de la evaluación, lo que lleva al planteamiento de las siguientes cuestiones: ¿Para quién se hace la evaluación? ¿Cómo aprenden las diferentes audiencias de los resultados obtenidos y/o del proceso de evaluación? ¿Cómo afecta este aprendizaje a la esfera política? En relación a este último aspecto, Weiss (1998) destaca que las decisiones acerca de una política o programa en un contexto democrático no tienen lugar en un contexto autocrático, sino que la evaluación tiene que tener en cuenta a grupos diferentes, cada uno de los cuales tiene algo que decir acerca del futuro de la inter138 vención pública evaluada. 
Este fue nuestro punto de partida teórico a la hora de enfocar nuestro trabajo de evaluación intermedia del Plan de Desarrollo Rural Sostenible (PDRS) 2000-2006 del País Vasco. En consecuencia, desde el principio, nuestro principal objetivo fue incrementar la utilidad social de la evaluación en un contexto específico, el de la política de desarrollo rural en el País Vasco. Para lograr este objetivo, el equipo evaluador consideró que el mejor método de trabajo era introducir, desde el comienzo de la evaluación, un debate interno sobre dos cuestiones principales anteriormente ya mencionadas: los propósitos de la evaluación y sus actores protagonistas. En el primer caso, se negoció con los usuarios primarios de la evaluación y fue posible llegar a un consenso sobre los propósitos de la evaluación y sus usos: satisfacer y responder a las necesidades de información de las diferentes audiencias (decisores políticos, equipos de gestión y otros posibles usuarios de la evaluación). La evaluación debía estimular la formulación de sus propias preguntas de evaluación: aquellas que correspondieran a sus intereses, percepciones e interpretaciones acerca del PDRS, y sus dudas y preocupaciones acerca de las acciones evaluadas. Esta aproximación participativa debía constituir un modo de generar conocimiento y facilitar el aprendizaje.

En segundo lugar, se discutió sobre los potenciales destinatarios de la evaluación (“¿Para quién se hace la evaluación?”). En relación con esta cuestión, el equipo evaluador manifestó un interés especial en fomentar la participación activa de todos los potenciales usuarios. El objetivo era transformar la evaluación en "su" evaluación, donde fueran formuladas "sus" preguntas de evaluación y no únicamente aquellas elaboradas por la Comisión Europea. En este proceso, se decidió trabajar en estrecha colaboración con los usuarios primarios en un intercambio abierto de información, en el que cualquier stakeholder (o agente crítico) tenía la oportunidad de tomar parte en el debate. Esta colaboración debía ayudar a producir aprendizaje formal e informal, desmitificando el proceso de evaluación en sí mismo, clarificando su papel y mostrando las expectativas que podía ofrecer la evaluación. Un proceso que, en definitiva, debía generar confianza mutua.

Teniendo en cuenta todas estas cuestiones, el artículo presenta el modelo de evaluación utilizado y el impacto obtenido en relación con la utilización de los resultados y del propio proceso de la evaluación. Para ello, el artículo se compone de dos partes principales. En primer lugar, se analiza el contexto de referencia en el que se desarrolló la evaluación intermedia del PDRS (estructural, institucional y administrativo y, desde una perspectiva más amplia, el contexto sociopolítico de la evaluación). Además, esta primera parte describe la adecuación del modelo de evaluación participativa a los intereses y propósitos de la evaluación. En segundo lugar, se examina el alcance que la evaluación propuesta tuvo y el grado de utilización alcanzado desde la perspectiva de los diferentes stakeholders participantes. Esto es, las valoraciones obtenidas a partir de la realización de un cuestionario y de la información recopilada en las entrevistas en profundidad, los dos métodos utilizados para conocer el alcance de la evaluación y su uso. El uso de los resultados y procesos fue valorado en función de cuatro áreas principales de influencia: 1) la información suministrada, 2) la generación 
de conocimiento, 3) la mejora de la aplicación (mejora de los programas) y 4) el impacto en la política (mejora de las políticas rurales en el País Vasco).

\section{EL ENFOQUE PARTICIPATIVO EN EVALUACIÓN}

Para garantizar la sostenibilidad del desarrollo rural, la evaluación debe estar guiada, como el resto de las fases de programación de políticas, por los criterios de gobernanza aplicables a los programas de desarrollo sostenible, criterios tales como la participación de los diferentes agentes implicados, la obtención de consensos, la transparencia, o el logro de la eficacia y la eficiencia de los programas'.

En esta línea, y como ya se ha mencionado anteriormente, el objetivo de la evaluación intermedia del PDRS era doble: por un lado, generar información que facilitara y enriqueciera el proceso de discusión sobre los problemas, favoreciendo a la vez la capacidad para la acción y el aprendizaje colectivo ("empoderamiento") a través de la participación y la transparencia; por otro lado, incrementar el uso de los resultados de la evaluación, ayudando a la Administración Vasca a tomar nuevas decisiones acerca de las políticas futuras de desarrollo rural sostenible y de sus programas (contribuyendo a políticas más eficientes y eficaces). En este contexto, hacer un buen uso de la evaluación, garantizando una amplia difusión de sus resultados, facilitaría la compresión por parte de los agentes de las condiciones, procesos y restricciones a las que se hallan sometidas las actuaciones públicas, lo que estimularía la participación y la implicación en la ejecución y en la búsqueda de los objetivos del Programa. Siendo conscientes de la amplitud que presentaban ambos propósitos, la elección de un modelo de evaluación participativa resultó la propuesta más idónea para intentar alcanzar dichas metas.

Partiendo de la idea de que la evaluación se enmarca dentro de un proceso democrático (Murray, 2002; Green, 2006), como equipo evaluador, centramos nuestra atención en las relaciones entre el concepto de democracia y el modo en el que los diferentes actores participan en la evaluación. Desde diferentes disciplinas y tradiciones dentro del campo de la evaluación, existe un amplio abanico de aproximaciones relacionadas que han sido agrupadas bajo el paraguas de evaluación participativa. Si bien existen algunas diferencias entre estas aproximaciones, todas ellas comparten un compromiso con el desarrollo de un cambio o mejora que es interactivo, contextualizado y dirigido a la construcción de conocimiento. Esta aproximación ha dado lugar a la aparición de prácticas evaluadoras innovadoras, tanto desde un punto de

1 El Libro Blanco de la Gobernanza Europea (European Commission, 2001) establece cinco principios como base de la buena gobernanza: la apertura (en el sentido de mantener una comunicación más activa sobre las acciones y decisiones de la UE), la participación (de la ciudadanía en todas las fases del proceso), la responsabilidad (de las diferentes instituciones), la eficacia (producir los resultados buscados sobre objetivos claros) y la coherencia (de las medidas y actuaciones, que respondan a los problemas identificados). 
vista teórico como práctico (Connell et al., 1995; Fetterman, 2001; Green, 1998; Patton, 2008; Plottu y Plottu, 2009; Whitmore, 1998).

Esencialmente, la evaluación participativa parte del reconocimiento de que la evaluación se desarrolla dentro de una sociedad pluralista, lo que permite que la evaluación se edifique sobre las ideas, valores, percepciones y aspiraciones de los participantes a todos los niveles y a través de todo el proceso. En este sentido, Gregory (2000) destaca cómo la participación en la evaluación asegura que las personas estén al tanto de la racionalidad que deriva de la toma de decisiones, y que su implicación sea mayor a la hora de ponerla en marcha de forma eficaz y eficiente.

Por tanto, se trata de un modelo que no es impuesto desde fuera, sino que se forma gradualmente a través de la colaboración de todos los actores implicados y su participación activa en el proceso de evaluación. Este enfoque, en consecuencia, incrementa considerablemente las probabilidades de que los resultados alcanzados por la evaluación sean efectivamente utilizados para mejorar la actuación pública, ya que permite que los participantes en el programa se apropien del proceso de evaluación y de sus resultados, convirtiendo la actividad evaluadora en un aprendizaje que, en mayor o menor medida, les pertenece.

\section{CUADRO 1}

Evaluación convencional versus evaluación participativa

\begin{tabular}{|l|l|l|}
\hline ¿Quién? & Expertos externos. & \multicolumn{1}{|c|}{ Participativa } \\
\hline ¿Qué? & $\begin{array}{l}\text { Las necesidades informativas y los cri- } \\
\text { terios de la evaluación están pre-deter- } \\
\text { minados. Evaluación por objetivos. }\end{array}$ & $\begin{array}{l}\text { Los agentes participantes identifican } \\
\text { sus necesidades informativas y determi- } \\
\text { nan sus propios criterios de evaluación. } \\
\text { a'Cómo? } \\
\text { res, personal del proyecto, destinatarios } \\
\text { directos e indirectos y el equipo de eva- } \\
\text { luación como facilitador. }\end{array}$ \\
\hline $\begin{array}{l}\text { Centrada en la objetividad científica, } \\
\text { distancia del equipo evaluador del } \\
\text { resto de participantes. }\end{array}$ & $\begin{array}{l}\text { Los métodos de evaluación y sus resul- } \\
\text { tados son compartidos entre todos los } \\
\text { agentes participantes. Transparencia y } \\
\text { entendimiento común. }\end{array}$ \\
\hline ¿Cuándo? & $\begin{array}{l}\text { En general, una vez finalizado el pro- } \\
\text { yecto, a veces en el intermedio. }\end{array}$ & $\begin{array}{l}\text { Más frecuente a lo largo de la aplicación } \\
\text { del programa: una forma de evaluación } \\
\text { continua. }\end{array}$ \\
\hline ¿Por qué? & $\begin{array}{l}\text { Rendición de cuentas. Evaluación su- } \\
\text { mativa ¿debe continuar el proyecto? }\end{array}$ & $\begin{array}{l}\text { Evaluación formativa para generar accio- } \\
\text { nes de mejora. Aprendizaje continuo. }\end{array}$ \\
\hline
\end{tabular}

Fuente: Adaptación propia en base a Narayan-Parker 1993: 12. 
En este contexto, el objetivo de transformar la evaluación en un proceso participativo de aprendizaje colectivo y de empoderamiento para la acción, implica que la evaluación:

- Produce información, promueve y enriquece el proceso de discusión sobre las necesidades y los problemas.

- Genera conocimiento, transforma las ideas y alcanza un mejor entendimiento del proceso real de cambio.

- Tiene capacidad para modificar las políticas y formular nuevas acciones públicas que contengan una mejor adaptación a la realidad social circundante.

A pesar de sus ventajas potenciales, la evaluación participativa era (y es) un enfoque escasamente utilizado en el País Vasco en la evaluación de las políticas de desarrollo rural y regional de la Unión Europea. Una realidad evaluadora que entra en contradicción con la defensa que, desde la propia Comisión Europea, se hace a favor de procesos de planificación y diseño de políticas, donde la participación de los actores regionales se convierte en un elemento esencial de los nuevos enfoques de la política regional y rural, tal como luego se explicará (ver apartado 3).

En nuestra opinión, las ventajas que introduce este modelo de evaluación cuando se utiliza en las políticas de desarrollo rural son claras:

- La evaluación es entendida como un proceso de aprendizaje alrededor de la política evaluada desde la perspectiva de todos los stakeholders. Son precisamente los participantes reales de las políticas los que contribuyen a entender y aprender acerca de los procesos de cambio subyacentes al programa y al desarrollo de una nueva visión relacionada con la política evaluada. Por lo tanto, la evaluación se convierte en un ejercicio que favorece la aparición de un proceso de aprendizaje.

- Este proceso común de aprendizaje colectivo permite la creación de un marco de trabajo en el que la evaluación es utilizada para construir entendimiento y confianza mutua (trust) entre los stakeholders, directores, instituciones y evaluadores (Kuhlmann, 1998: 138). La evaluación participativa hace posible democratizar el proceso de construcción del conocimiento.

- La evaluación es utilizada para generar un conocimiento útil para los implicados en el proceso de cara a alcanzar sus objetivos a corto y largo plazo. En una sociedad pluralista, donde existen multiplicidad de puntos de vista y perspectivas, no es posible ni deseable esperar obtener una medida objetiva exacta del impacto de la política, como verdad incuestionable (Díez, 2002; Guba y Lincoln, 1989; Patton, 2008; Suárez Herrera et al., 2009). Además, cuando el 142 objeto es evaluar políticas complejas, con un amplio número de metas, el ob- 
jetivo de la evaluación debe ser crear conocimiento práctico, en vez de juicios mecánicos relativos a los resultados, y la atención debe centrarse continuamente en los procesos de aprendizaje.

- Esta creación de conocimiento es, al mismo tiempo, un facilitador para la acción y movilización rural, que estimula la capacidad de los gobiernos regionales, instituciones comunitarias y organizaciones en general, para resolver los problemas pertinentes (Finne et al., 1995: 12), lo que contribuye a una mejora de la gobernanza, fundamental para el éxito de los programas. En este contexto, la evaluación participativa favorece el aprendizaje para la acción, dado que el proceso de evaluación es utilizado para impulsar la acción dirigida a la mejora de la política.

- La evaluación participativa, por lo tanto, hace posible reforzar e incrementar el poder de los participantes para resolver sus problemas económicos y sociales. La evaluación se entiende como un proceso catalizador del cambio que combina la creación de conocimiento y, a través del aprendizaje, facilita la movilización para la acción. En definitiva, la evaluación participativa debe ser conceptualizada como un modo de desarrollar conocimiento, facilitar el aprendizaje y capacitar a los diferentes stakeholders a resolver los posibles desafíos de la región.

La aplicación de un modelo de evaluación participativa a la evaluación intermedia del Plan de Desarrollo Rural Sostenible (PDRS) 2000-2006 supuso una ruptura importante con la cultura evaluadora previa. En efecto, el enfoque participativo abría un nuevo escenario en la práctica de la evaluación en el País Vasco, ya que, hasta ese momento, las evaluaciones de desarrollo rural se habían realizado casi exclusivamente con el objetivo de cumplir con los compromisos formales adquiridos con la Comisión Europea.

Pero además, y lo que es más importante, este modelo de evaluación significó un paso adelante en el objetivo de diseñar una evaluación que fuera socialmente útil, para las instituciones implicadas en el diseño de la política de desarrollo rural. Desde esta perspectiva, la evaluación se convirtió en un ejercicio dirigido a satisfacer las necesidades de información de los participantes en el programa, así como destinado a promover su participación activa en el proceso de evaluación (Stame, 1999; Díez, 2002; Suárez Herrera et al., 2009).

Aquí, sin embargo, es preciso hacer algunas referencias explícitas, a quiénes fueron los principales agentes que participaron en la evaluación intermedia del PDRS. La novedad del enfoque evaluador requería actuar con prudencia a la hora de proceder a la apertura del proceso de evaluación. Por esta razón, se decidió que los participantes debían ser aquellos grupos que se encontraban más cercanos al ámbito político y técnico del programa evaluado, es decir, los usuarios primarios de la 
evaluación. Este colectivo abarcaba a los decisores políticos, los equipos de gestión y/o operativos y otros agentes críticos tales como expertos en medio rural, asociaciones sectoriales, etcétera².

Durante todo el proceso de la evaluación, asegurar la participación activa de estos stakeholders se convirtió en uno de los principales desafíos de la evaluación intermedia del PDRS 2000-2006 del País Vasco, siendo considerado un elemento necesario e imprescindible a la hora de fortalecer la utilidad de los resultados ${ }^{3}$. Al igual que otros autores (Patton, 2008; Cousins y Whitmore, 1998; Burke, 1998; Forss et al., 2002; Díez, 2001; Suárez Herrera et al., 2009; Subirats, 2006), estimamos que la implicación de los stakeholders en el proceso de evaluación comporta un incremento en la utilización de sus resultados, de modo que cuanto mayor sea ésta, se incrementara igualmente la posibilidad de poner en práctica los resultados y las recomendaciones de la evaluación.

\section{EVALUACIÓN Y DESARROLLO RURAL: DEL CONTEXTO EUROPEO AL ÁMBITO REGIONAL}

\subsection{El contexto evaluador en Europa: breves apuntes}

La creciente relevancia de la política de cohesión social y económica dentro de las prioridades estratégicas de la Unión Europea ha servido para fortalecer la necesidad de obtener mayor transparencia en el uso de los fondos públicos comunitarios y garantizar la eficacia y eficiencia de los recursos de los Fondos Estructurales. La forma más adecuada para conseguir estos objetivos ha sido la incorporación de la evaluación como requisito a la hora de acceder y gestionar estos Fondos. En el caso de España, su ingreso en la Unión Europea supuso la introducción de la evaluación en el ámbito de las políticas públicas, lo que ha dado lugar a cambios significativos en el campo de la evaluación (Viñas, 2009).

En efecto, el aumento significativo de la actividad evaluadora y su desarrollo en Europa en los últimos años se deriva del progresivo interés mostrado por la Comisión Europea por esta actividad y la presión ejercida sobre los Estados miembros para introducir la evaluación de sus políticas financiadas por los Fondos Estructurales.

Esta preocupación de la Comisión por institucionalizar la evaluación se ha canalizado a partir de dos direcciones complementarias: a través de la creciente presencia de la evaluación en los Reglamentos de los Fondos Estructurales hasta conseguir in-

2 En definitiva, el criterio para la selección de participantes fue un criterio operativo, lo que sitúa al enfoque evaluador aplicado dentro de la corriente de evaluaciones participativas denominadas "Practical Participatory Evaluation" (P-PE) (Cousins y Withmore, 1998).

3 Al igual que Weiss, destacamos la relevancia de la participación en evaluación. Tal y como la autora apunta: "El mejor modo que conocemos hasta el momento para estimular el uso de la evaluación 144 es a través de la implicación de los usuarios potenciales a la hora de definir el estudio e interpretar los resultados, y a través de la diseminación continuada de éstos mientras dura el estudio" (1998: 30). 
corporar la evaluación dentro de sus mecanismos reguladores y, por otro lado, a partir de los progresos realizados en el ámbito metodológico.

Con el objetivo de armonizar conceptos y extender las herramientas y técnicas de evaluación, la Comisión puso en marcha, en el año 1994, el Programa MEANS (Métodos para Evaluar las Actuaciones de Naturaleza Estructural). La finalidad de este programa era mejorar las metodologías utilizadas en la evaluación de los Fondos Estructurales y desarrollar una cultura de evaluación en Europa ${ }^{4}$. Entre sus cometidos, se encontraba mejorar la calidad de las evaluaciones, reforzar la credibilidad de los estudios de evaluación frente a las diferentes instancias europeas, y aumentar la utilidad de estas evaluaciones (Bachtler y Michie, 1995: 747).

Para lograr este objetivo, el programa MEANS publicó una serie de Cuadernos Metodológicos ("MEANS Handbooks"), a los que seguiría en 1999 una Guía de evaluación (Centre for European Evaluation Expertise, 1996). La relevancia alcanzada por la colección MEANS impulsó a la Comisión a actualizar y revisar su contenido, lo que tuvo como resultado la publicación de una nueva guía (The Guide) a finales de 2003, una publicación que, desde ese año, está disponible en formato electrónico, lo que permite su revisión y actualización continua5. A través de esta nueva guía, la Comisión, consciente de los avances surgidos en la práctica evaluadora de los Fondos Estructurales a lo largo de los últimos años, promueve la utilización de nuevas herramientas de evaluación, dotando de especial interés a las aproximaciones participativas.

Sin duda, todas estas actividades y esfuerzos han contribuido a una creciente calidad de las evaluaciones. Se ha producido una mejora de las metodologías utilizadas y de la armonización de conceptos, así como se ha contribuido al desarrollo de un marco global y coherente y a la elaboración de un discurso común (Díez et al., 2006: 203). En este sentido, la guía se ha convertido en un manual de referencia de gran valor por su capacidad para integrar y presentar diferentes perspectivas y enfoques de evaluación, mostrando alternativas y dejando libertad para adoptar elecciones metodológicas adecuadas a cada situación evaluativa.

Sin embargo, y a pesar de los avances logrados, un estudio realizado por la Comisión en año 2004 (European Policy Evaluation Consortium, 2005) evidenciaba las carencias de la práctica evaluadora de los Fondos Estructurales. Entre estas limitaciones se destacaba el limitado trabajo de campo que presentaban la mayoría de las

4 La práctica evaluadora de los Fondos Estructurales durante los primeros años, puso en evidencia la existencia de deficiencias muy importantes relativas a los modelos y métodos adoptados en la actividad evaluadora, existiendo un gran vacío en relación a las orientaciones metodológicas dirigidas al diseño y realización de las evaluaciones. Derivado de esta práctica, las evaluaciones en estos años se van a caracterizar por su baja calidad, una utilidad muy reducida y un nivel técnico que estaba en estrecha relación con la experiencia nacional y la propia cultura de evaluación de cada país miembro (Díez et al., 2006: 188).

5 En línea: <http://ec.europa.eu/regional_policy/sources/docoffic/working/sf200o_en.htm.>. 
evaluaciones, con escasas referencias a la percepción de los gestores y beneficiarios de los programas. Además, el informe hacía referencia explícita al limitado uso de la evaluación, reflejando cómo los resultados de las evaluaciones no eran utilizados para mejorar y reformular los programas. Dentro de este ámbito, la Comisión incidía así mismo en el escaso uso de la evaluación a nivel político, siendo su aportación a los debates democráticos locales y nacionales muy baja.

Por otro lado, atendiendo a la política de desarrollo rural, se observa cómo la situación anteriormente descrita refleja igualmente el escenario en el que se ha desenvuelto su evaluación, siendo las evaluaciones diseñadas y dirigidas desde Europa casi la única práctica de evaluación a la que ha estado sometida la política de desarrollo rural. Además, el limitado interés por la evaluación de este tipo de políticas entre la comunidad evaluadora ha favorecido una situación en la que prácticamente la única información de que se dispone procede de las evaluaciones oficiales. Una información útil y muy valiosa acerca de las actuaciones realizadas y los resultados alcanzados, pero en la que apenas existe información de lo que ocurre en el terreno, siendo las aproximaciones participativas muy limitadas.

Esta última cuestión resulta clave en el debate acerca de la evaluación de las políticas de desarrollo rural, ya que, desde nuestra perspectiva, la evaluación de este tipo de políticas debe de ser entendida desde una perspectiva participativa. La propia naturaleza de estas políticas, diseñadas a través de modelos de participación de abajo hacia arriba (o "bottom- up"), conduce a un cuestionamiento lógico del modelo de evaluación utilizado hasta ahora, e "invita a buscar modos alternativos de entender el rol y la naturaleza de las evaluaciones” (Ray, 2000: 2) y de su aplicación (Rist y Stame, 2006).

Del mismo modo, y como se verá a continuación, la descentralización institucional en la que se desenvuelven las políticas de desarrollo rural favorece la utilización de este modelo evaluador, siendo a nivel regional y local donde la evaluación participativa puede ser puesta en práctica más fácilmente. Evaluadoras como Stame han apoyado esta aproximación. Esta autora sostiene que la evaluación de las políticas de desarrollo regional debería hacer un mayor uso de los enfoques participativos (1999: 105) y tomar en consideración los puntos de vista de los diferentes actores implicados a la hora de diseñar las evaluaciones, seleccionar su metodología y el contenido propio de la evaluación (Stame, 2006a).

\subsection{La política de desarrollo rural europea y su aplicación en el País Vasco}

La política de desarrollo rural europea, denominada "segundo pilar" de la Política Agraria Comunitaria (PAC), es una de las principales herramientas financieras de las que dispone la Comisión Europea para el desarrollo y sostenimiento socioeconómico de las zonas rurales más desfavorecidas. En su evolución en los últimos años, la política de desarrollo rural en Europa ha ido evolucionando desde una visión estricta-

146 mente agraria hacia una idea de desarrollo mucho más amplia. 
Tras años de discurso agrario, el desarrollo de las zonas rurales ha ido ampliando su ámbito de actuación, y la noción de desarrollo rural ha ido evolucionando hacia una visión integral del desarrollo, marcada por la diversificación de actividades productivas más allá del sector primario ("desarrollo multisectorial”). Esta multiplicidad de actividades se hace extensible al propio sector agrario, en una apuesta por diversificar sus actividades $y$, por ende, sus funciones. Se trata de fomentar un tipo de agricultura que produzca alimentos, pero que además cumpla otras funciones ("multifuncionalidad de la agricultura"). Una agricultura, en definitiva, que supere el aspecto puramente productivo y contribuya a conservar un espacio socioeconómico viable, protegiendo el entorno paisajístico y el medio ambiente.

Esta evolución en el concepto del desarrollo rural se puede observar también cuando se examinan los planes de desarrollo aprobados en las diferentes zonas rurales europeas. Este es el caso, por ejemplo, del Plan de Desarrollo Rural Sostenible 2000-2006 del País Vasco, pieza fundamental del desarrollo rural en esta Comunidad durante estos años y primer ejemplo de una programación basada en una concepción de desarrollo rural integral.

\title{
CUADRO 2
}

\section{EI PDRS del País Vasco 2000-2006: un breve resumen}

\begin{abstract}
Los objetivos principales del PDRS 2000-2006 eran: 1) consolidar la actividad agraria como integrante significativo del tejido socioeconómico rural, 2) apoyar la viabilidad de las explotaciones agrarias, impulsando una mayor implicación de los activos agrarios en la cadena de valor, con el fin de lograr una mayor generación de valor añadido y 3) aumentar la competitividad de las zonas rurales, mediante la contribución a la generación de empleo.

El ámbito territorial del Plan se extendía a un total de 113 municipios rurales que suponían más de la mitad de la superficie de la CAPV (52\%), pero que apenas representaban el $3,7 \%$ de la población vasca (76.869 habitantes) en 2001.

Este Plan estaba cofinanciado por las Administraciones vascas (Gobierno Vasco y las tres Diputaciones Forales) y el FEOGA-Garantía. El plan financiero preveía un gasto público para el periodo de 235,8 millones de euros, de los cuales el FEOGA aportaba 121,2 millones $(51,4 \%)$. Las actuaciones se articulaban en torno a nueve medidas: inversiones en explotaciones agrarias (13,4\% del gasto público), instalación de jóvenes agricultores (0,8\%), formación ( $1 \%)$, cese anticipado (2,2\%), indemnizaciones para explotaciones en zonas desfavorecidas ( $8 \%)$, medidas agroambientales (13\%), transformación y comercialización de productos agrarios (23,9\%), selvicultura $(16,4 \%)$ y adaptación y desarrollo de las zonas rurales $(21,3 \%)$.
\end{abstract}

Fuente: Elaboración propia.

En efecto, derivado de esta visión renovada del desarrollo rural, el PDRS se convirtió en un claro exponente de un programa integrado y, además, muy complejo: constaba de 9 medidas, más de 35 líneas de actuación y una media de 7.000 be- 
neficiarios potenciales anuales ${ }^{6}$. Además, se trataba de un Plan en el que intervenían un amplio abanico de instituciones a distinto nivel: desde el ámbito europeo al local (Fondos Estructurales-Fondo Europeo de Orientación y Garantía Agrícola (FEOGA), el Gobierno Vasco, Diputaciones Forales, municipios, sindicatos, representantes de las comunidades locales como ayuntamientos, asociaciones etc.). También participaban otras agencias públicas, como las Oficinas Comarcales Agrarias y las Asociaciones de Desarrollo Rural7, que eran las encargadas de la difusión de las medidas y mantenían un contacto más directo con los potenciales colectivos de beneficiarios/as.

En consecuencia, el modelo de evaluación participativa propuesto para su evaluación debía desarrollarse dentro de un escenario complejo y multinivel, donde las necesidades de coordinación entre las diferentes administraciones implicadas adquirían una relevancia crucial. En este sentido, la evaluación debía tener en cuenta este contexto institucional de múltiples agentes a la hora de definir la potencial contribución de la evaluación a generar, y difundir conocimiento sobre las actuaciones públicas y asegurar su difusión y circulación entre los distintos niveles administrativos (incluida la Comisión Europea), de forma que mejorara la gobernanza de las políticas de desarrollo rural.

\subsection{La evaluación intermedia del PDRS del País Vasco 2000-2006}

En este contexto, a finales del año 2001, el Departamento de Agricultura del Gobierno Vasco como organismo responsable de la elaboración y ejecución del PDRS 2000-2006, decidió contactar con un equipo multidisciplinar de la Universidad del País Vasco con el objetivo de iniciar los trabajos relativos al establecimiento y organización de un sistema de evaluación que le permitiera conducir las evaluaciones intermedia (2003) y ex post (2008) de acuerdo a las directrices requeridas por la Comisión Europea.

A partir de este momento, se inició un proceso de trabajo marcado por la necesidad de mejorar la calidad y utilidad social de las evaluaciones de las políticas de desarrollo rural. Con este doble reto en mente, el equipo evaluador decidió proponer el uso de un modelo de evaluación participativa, con el objetivo final de incrementar la utilización pública de los resultados y del proceso de evaluación. La elección de esta aproximación evaluadora, en un intento por diseñar una evaluación que fuera socialmente útil, suponía romper con la cultura de evaluación precedente ${ }^{8}$, tanto para las

6 La amplitud de aspectos que recogía el Plan quedaba también reflejada en la variedad de sus beneficiarios potenciales. Desde personas físicas, jurídicas y/o asociaciones (de desarrollo rural, cultural o sectorial), hasta entidades públicas como Ayuntamientos, Mancomunidades y hasta las propias Diputaciones Forales, eran, todos ellos, posibles beneficiarios directos del mismo Plan.

7 En la CAPV existen un total de 21 Oficinas Comarcales Agrarias (OCAS) y alrededor de 16 Asociaciones de Desarrollo Rural.

8 El enfoque participativo en evaluación abre un nuevo escenario en la práctica de la evaluación en nuestro contexto, dado que salvo determinadas excepciones, las evaluaciones de desarrollo rural reali- 
instituciones regionales implicadas en la gestión del desarrollo rural, como para la propia comunidad rural.

Como ya se indicaba en el apartado 2, el diseño de una evaluación participativa respondía a la necesidad de incrementar la utilización de los resultados de la evaluación y, para ello, la participación de los stakeholders en el proceso de evaluación fue el enfoque metodológico adoptado. Al mismo tiempo, se intentó familiarizar a los stakeholders con el proceso y los resultados de la evaluación, como forma de contribuir a generar una mayor cultura evaluadora, considerando que "un mejor entendimiento de los beneficios de estar implicado en un proceso de evaluación supone claramente un progreso en el pensamiento evaluativo" (Valovirta, 2002: 64).

Desde este nuevo enfoque, el equipo de evaluación buscaba realizar un ejercicio evaluador capaz de cumplir las siguientes funciones:

1. Generar unos resultados útiles a la hora de orientar a la administración vasca en la toma de decisiones en materia de política de desarrollo rural, mejorando así la gobernanza de los procesos de desarrollo rural.

2. Satisfacer las necesidades de información de la Comisión Europea, realizando una valoración global de los resultados obtenidos en los planes cofinanciados por el Fondo Europeo de Orientación y Garantía Agrícola.

3. Democratizar la evaluación y hacerla así transparente.

4. Contribuir a generar cultura evaluadora en nuestro entorno.

El primer encargo para el equipo evaluador (en el año 2001) consistió en diseñar una Guía metodológica para la elaboración de la evaluación intermedia y ex post del PDRS 2000-2006. El diseño de esta Guía se extendió hasta finales del año 2002 y, junto a otras tareas, supuso también abordar una revisión completa de los sistemas de indicadores de seguimiento. El segundo encargo fue la elaboración de la evaluación intermedia 2000-2002, un trabajo que se llevó a cabo durante todo el año 2003. Finalmente, en el mes de mayo del 2004 se realizaron una serie de presentaciones de los resultados obtenidos en la evaluación con objeto de difundir el informe de evaluación a una audiencia más amplia. Al final de dichas sesiones, a todas aquellas personas que habían tomado parte en el proceso de la evaluación intermedia, se les entregó un cuestionario semi-abierto elaborado por el equipo evaluador con la finalidad de conocer los resultados, el alcance y los efectos del modelo de evaluación utilizado.

\footnotetext{
zadas hasta ese momento en el País Vasco habían tenido como único objetivo cumplir los requisitos exigidos por Bruselas. A lo largo de nuestro trabajo hemos podido comprobar cómo los resultados de evaluaciones anteriores no habían sido difundidos, por lo que apenas tenían repercusiones sobre los gestores de las ayudas, que desconocían los informes de las evaluaciones previas, aunque recordaban que alguien les había entrevistado con este objetivo.
} 
Por tanto, fue un proceso de trabajo que se extendió a lo largo de casi dos años y medio (evidentemente, con diferencias de intensidad en relación al esfuerzo y a las tareas realizadas) y en el que, además de los componentes del equipo de evaluación, participaron alrededor de 60 personas. La larga duración de la evaluación estuvo directamente relacionada con la complejidad del Plan evaluado, su naturaleza interinstitucional y su elevado grado de descentralización, tres elementos que condicionaron y marcaron la intensidad de los ritmos de trabajo.

Durante toda la evaluación, se intentó conseguir una participación activa de los diferentes implicados. Así, los usuarios potenciales de la evaluación participaron, junto con el equipo evaluador, en el desarrollo de numerosas tareas evaluadoras: desde la delimitación de criterios y preguntas de evaluación y la selección de los indicadores y la búsqueda de fuentes de información, hasta la revisión e interpretación de resultados y la discusión de las conclusiones y de las recomendaciones.

\section{ANALIZANDO LA UTILIDAD DE LA EVALUACIÓN DEL PDRS}

\subsection{Posibles usos de los resultados y el proceso de evaluación}

Existe una amplia literatura de evaluación donde se reflexiona y se analiza en torno a la utilidad de la evaluación. Los diferentes usos de la evaluación y su papel en la toma de decisiones se describen en trabajos y artículos de numerosos autores (Chelimsky, 2006; Feinstein, 2002; Patton, 2001; Patton, 2008; Stame, 2006b), entre los que destacan en particular las aportaciones realizadas por Carol Weiss (1987; 1998; Weiss y Bucuvalas, 1980).

Es precisamente un artículo de Weiss y Bucuvalas escrito en 1980 el que nos sirvió para estructurar nuestro análisis de la utilidad de los resultados y el proceso de la evaluación. De acuerdo con estas autoras, los participantes juzgan la investigación social de acuerdo a dos marcos de referencia. En primer lugar, aplican un "test de la verdad" ("truth test"), a través del cual se valora la calidad de la investigación y su conformidad con las expectativas y conocimiento previo. En un segundo momento, se consideran las implicaciones que la información facilitada tiene para el desarrollo de posibles acciones políticas futuras, aplicando un "test de utilidad" ("utility test") (Weiss y Bucavalas, 1980).

Teniendo como marco de referencia estas dos pruebas, el equipo de evaluación diseñó un cuestionario semi-estructurado con el propósito de recoger información que sirviera para analizar la relevancia, la calidad y la utilidad de la evaluación. En total, se cumplimentaron 48 cuestionarios que fueron respondidos por los distintos stakeholders en las sesiones de difusión final del informe de evaluación. Además, tal y como se apuntaba al inicio, los resultados arrojados por los cuestionarios fueron completados con información cualitativa a partir de la realización de una serie de entrevistas en 150 profundidad dirigidas a un número reducido de los participantes en el proceso de eva- 
luación. Para su análisis y sistematización, los efectos identificados se agruparon en dos áreas de influencia o impacto de los resultados y el proceso de la evaluación:

- Un uso de naturaleza conceptual (educativo o de aprendizaje). Para ello, se utilizaron tres criterios de valoración: la información suministrada, la generación de conocimiento y el acuerdo con la metodología aplicada como forma de estimar la relevancia y rigurosidad de la evaluación. Estos tres indicadores permitían desarrollar el "truth test".

- Un uso de naturaleza instrumental relacionado, por lo tanto, con la propia mejora del programa evaluado. Aquí, se utilizaron dos criterios de valoración: la mejora de la aplicación y el impacto potencial sobre la política. De esta forma, se establecía nuestro "utility test".

A continuación, el apartado siguiente resume brevemente los resultados arrojados por los cuestionarios?

\subsection{Sinopsis de las valoraciones obtenidas en los cuestionarios}

En la mayoría de las preguntas incluidas en el cuestionario, se requería a los actores participantes que otorgasen una puntuación en una escala del 1 al 4 (de menor a mayor) a las cuestiones en las que se desarrollaban los diferentes criterios de valoración.

El primer criterio era su valoración acerca de la información suministrada, un criterio donde se les pedía valorar la capacidad de la evaluación a la hora de recoger y presentar información completa y actualizada, comparar sus resultados con los de otros ámbitos, diagnosticar problemas, identificar áreas de mejora y ofrecer una síntesis ajustada de la situación, así como para dar una visión global de la política de desarrollo rural. La valoración media alcanzada acerca de todas estas cuestiones era de 2,8.

El segundo criterio era la generación de conocimiento. Se trataba de comprobar si la evaluación había ayudado a la aparición de nuevas ideas, o más bien, les había permitido confirmar ideas ya pre-existentes o, incluso, corregir apreciaciones previas que la evaluación no refutaba. Aquí, la evaluación sobre todo servía para confirmar hechos $(3,0) y$, en menor medida, para generar nuevos conocimientos $(2,1)$.

El tercer criterio trataba de apreciar su acuerdo con la metodología. La puntuación obtenida fue en general alta, alcanzando una media de 3,0 puntos en todas las cuestiones relacionadas con los métodos utilizados, los indicadores, la cobertura de

9 El Anexo ofrece información detallada de las puntuaciones obtenidas en cada pregunta incluida bajo cada criterio de evaluación. 
temas y la dinámica de trabajo. Fue en este último aspecto, sin embargo, donde se obtuvo la puntuación más baja de este grupo $(2,7)$.

El cuarto criterio intentaba averiguar cuál era la utilidad de la evaluación en cuanto a su capacidad para mejorar la aplicación y el funcionamiento de las medidas. En esta área, las puntuaciones obtenidas fueron sensiblemente inferiores a las de los criterios anteriores. Con 2,5 puntos de valoración aparecían las respuestas relativas a comunicar y compartir información y experiencias, reflexionar sobre el trabajo diario, así como cuestiones relacionadas con la mejora de los flujos de información interna y el seguimiento. La puntuación descendía hasta 1,9 puntos en cuestiones relativas a la contribución de la evaluación a mejorar la gestión.

Finalmente, el quinto criterio valoraba el impacto potencial de la evaluación sobre la política de desarrollo rural, es decir, si ésta había ayudado a reflexionar sobre el futuro de la política, a diseñar nuevas medidas y a introducir cambios en las medidas. En estas tres cuestiones, de nuevo la puntuación obtenida fue alta, oscilando alrededor de los 3 puntos. De nuevo, sin embargo, la valoración bajaba ante cuestiones relativas a cambios de funcionamiento y cambios de gestión. Por último, se incluía una valoración global sobre el nivel de relevancia, donde se alcanzaba un puntuación de 3,1 y del nivel de satisfacción global, que era de 3 puntos.

\subsection{Resultados sobre el uso de la evaluación en el marco de la gobernanza}

En primer lugar, el análisis realizado demostró que la evaluación había contribuido a incrementar la relevancia y significación de los resultados obtenidos. En este sentido, se puede afirmar que la información suministrada era muy apreciada. Tal y como se pudo contrastar en las entrevistas en profundidad, el informe de evaluación se había convertido en un documento de referencia para los agentes, llegando a audiencias cada vez más amplias. Era un documento que ayudaba al debate y favorecía la discusión basada en evidencias contrastadas y conclusiones bien argumentadas. Por lo tanto, se valoraba positivamente la transparencia sobre los resultados obtenidos en la evaluación.

En segundo lugar, también se había facilitado una mayor apropiación por los propios usuarios de los resultados de la evaluación. Las conclusiones y las recomendaciones eran transparentes, conocidas por todos los actores del proceso, derivadas del proceso lógico de razonamiento de la evaluación, y difundidas con posterioridad a audiencias más amplias. De esta manera, el modelo de evaluación adoptado había contribuido a visualizar la política de desarrollo rural y la red de agentes, y su papel económico y social en la realidad de la economía vasca, una realidad marcada siempre por la preponderancia de la vida industrial. Así lo percibían por lo menos los actores de la evaluación, que pensaban que la evaluación había ayudado a que su trabajo 152 y su labor a favor de la región fueran más conocidos. 
En tercer lugar, la evaluación había contribuido a promover e impulsar una mayor cultura de evaluación. En particular, la metodología utilizada había ayudado a introducir nuevas prácticas y a comenzar a desarrollar una cierta cultura evaluadora: "las evaluaciones no se habían hecho así antes. Hemos tenido que aprender" decía uno de los actores en el cuestionario. Incluso el mero hecho de leer el informe de evaluación o escuchar las presentaciones familiarizaba a los oyentes con el lenguaje y los métodos de la evaluación. La evaluación en sí misma comenzaba a ser valorada como forma de aprendizaje y desarrollo de nuevas capacidades.

En cuarto lugar, se pudieron detectar fallos y carencias del propio proceso de participación, que afectaban tanto a sus métodos como a la selección de los participantes. Así, por ejemplo, los actores, una vez finalizado el proceso de la evaluación, demandaban una dinámica de trabajo más grupal: más grupos de discusión y más reuniones sectoriales. El proceso de la evaluación había sido en gran parte un proceso muy individualizado, basado en entrevistas en profundidad y en el trabajo con los actores uno a uno. Sin embargo, los participantes demandaban más trabajo en grupo, con una dinámica dirigida a incrementar el flujo de información entre los diferentes participantes, tanto de diferentes áreas de trabajo (agraria-forestal, por ejemplo) como de diferentes territorios. También, se reclamaba una mayor apertura del proceso de la evaluación, una apertura dirigida hacia la inclusión de los colectivos de beneficiarios como agentes activos de la evaluación. Esta demanda se expresaba en términos como "deseamos conocer la opinión de los administrados". Una idea que aparecía entre los comentarios de un buen número de cuestionarios y en las entrevistas con los usuarios participantes.

En quinto lugar, y en relación con la aplicación directa de los resultados, el grado de satisfacción de los participantes era más bien bajo. Los actores reclamaban incluir aspectos más operativos y de gestión interna de las medidas. Esto es, "dirigir la evaluación hacia aspectos más prácticos". Es así como expresaban esta insatisfacción que igualmente se puso de manifiesto en las reuniones de difusión final. Esta insatisfacción provenía, fundamentalmente, de los técnicos y, en particular, de los equipos comarcales, muy preocupados por la necesidad de flexibilizar mecanismos y de reducir la parte más burocratizada de su trabajo para agilizar el desarrollo de sus tareas diarias de gestión y facilitar su atención a los colectivos beneficiarios, al tiempo que demandaban una simplificación de los procedimientos.

En último lugar, quizás una de las cuestiones más interesantes de las conclusiones obtenidas en este análisis sea el elevado impacto potencial que los actores otorgaban a la evaluación, es decir, su capacidad para modificar el diseño de la política de desarrollo rural y el propio programa y sus medidas de actuación de cara al futuro. Sin embargo, este resultado positivo puso también en evidencia otra de las debilidades que se habían ido producidiendo a lo largo del proceso de participación, y que era así expresada por uno de los participantes: "A ver si influye en nuestros políticos. Nosotros somos simples gestores que no tenemos poder de decisión, aún cuando sí de sugerencia". 
Y es que, en efecto, a pesar de que, durante todo el proceso de evaluación, el equipo evaluador realizó numerosos esfuerzos por involucrar de forma activa a todos los usuarios potenciales de la evaluación, es decir, decisores políticos, equipos de gestión, técnicos comarcales, expertos y/o otros agentes sociales, el éxito real alcanzado no fue el mismo en todos los niveles. Concretamente, el nivel de participación de los decisores políticos (Directores, Diputados de Agricultura, Viceconsejeros, etc.) fue escaso. Esto no quiere decir que los decisores políticos no estuvieran informados en todo momento de los avances de la evaluación, o no tuvieran acceso a todos los documentos sobre los progresos que se iban produciendo (prueba de ello fueron los comentarios enviados al equipo evaluador). Pero, en la práctica, su participación en las dinámicas de trabajo desarrolladas a lo largo de la evaluación fue más bien escasa, constituyendo más una excepción que la norma y el nivel de permeabilidad "de abajo hacia arriba" de los flujos de información conseguido no fue muy elevado. Ésta fue, sin duda, una importante limitación del proceso en términos de gobernanza.

Además, la participación de los decisores políticos también se vio afectada por otros problemas importantes de señalar como, por ejemplo, la pluralidad de instituciones implicadas, lo que multiplicaba por cuatro el nivel de interlocución necesario para implicar activamente a los decisores políticos. Esta desmembrada unidad central estuvo sometida, además, a los ciclos políticos propios de cada nivel administrativo, de manera que el proceso de la evaluación coincidió con elecciones locales que tuvieron como resultado un cambio de Diputados provinciales y, por consiguiente de directores, cambio que se produjo a mediados del año 2004 en todas las Diputaciones Forales.

\section{REFLEXIONES FINALES}

El objetivo de este artículo ha sido analizar y valorar el modelo de evaluación participativa puesto en práctica en la evaluación intermedia del PDRS vasco y, de manera específica, comprobar si la evaluación había contribuido a incrementar el uso de los resultados, así como llegar a hacer útil el propio proceso, ambos en el marco de la gobernanza de la política regional europea en el País Vasco. Un segundo propósito subyacente al diseño de esta evaluación era incrementar la cultura evaluadora de nuestro entorno.

A lo largo de estas páginas, hemos querido destacar las luces y las sombras de nuestro trabajo con el objeto de poder mejorar nuestra práctica como evaluadores y extraer lecciones de la experiencia adquirida a lo largo de este trabajo. La puesta en práctica de la participación en evaluación no es una tarea fácil. Existen obstáculos al proceso de participación que lo convierten en un trabajo exigente y complejo. En este sentido, ya hemos descrito cómo el proceso de la evaluación fue largo en el tiempo (dilatándose durante algo más de tres años), y costoso, en cuanto a la dedicación exigida al equipo de evaluación y también, en menor medida, a los stakeholders, con alti-

154 bajos a lo largo de su dinámica de trabajo, y en relación a su capacidad para generar 
conocimiento, pero creemos que el proceso fue a todas luces satisfactorio en cuanto a los resultados obtenidos y a su utilidad.

Además, las actitudes de los participantes hacia el proceso de evaluación puesto en marcha y su voluntad de trabajar en colaboración con el equipo evaluador, estuvo fuertemente afectada y condicionada por un número amplio de factores externos. Entre los obstáculos de tipo social y cultural, tenemos que destacar la poco habitual cultura de planificación en las administraciones vasca y española, lo que se traduce en un entramado institucional marcado por una planificación intervencionista y una escasa, si no existente, cultura de evaluación y de participación.

El análisis mostró asimismo el contexto atomizado en el que se ha desenvuelto la evaluación, en el que están implicadas numerosas instituciones y actores, lo que determina e influye en el proceso de evaluación y puede causar dificultades cuando ponemos en marcha una evaluación participativa. Esta diversidad queda claramente reflejada en el modo en el que se comportan los diferentes actores, y la manera en la que reaccionan cuando se enfrentan a un proceso participativo. Autores como Patton (1997) lo han definido como el factor personal, siendo determinante no sólo para alcanzar un alto grado de participación, sino también para comprobar si la evaluación tiene la capacidad de convertirse también en un proceso de aprendizaje colectivo (Patton, 2001 y 2008; Fetterman, 2001; Green 1998; Torres et al., 2005).

Sin embargo, a pesar de las dificultades de partida descritas, la evaluación consiguió alcanzar un nivel de participación elevado, especialmente entre los equipos de gestión, expertos y agentes sociales. Frente al uso retórico del que en ocasiones es objeto la participación en la planificación y en la evaluación, consideramos esta alternativa como una de las más válidas en la evaluación de la política de desarrollo rural. En nuestro caso, se consiguió una activa participación de los actores en el proceso de evaluación, siendo muy interesante la manera en que el control de la evaluación se ha ido desplazando de manera que los participantes se hacen con el control junto al equipo de evaluación de muchas de las tareas realizadas. Esta cuestión de quién toma las decisiones está estrechamente relacionada con el aprendizaje individual ya que "el aprendizaje y acumulación de experiencia tiene un impacto en el empoderamiento" (Themessl-Hubber y Grustch, 2003: 109). Por consiguiente, la evaluación ha demostrado ser una herramienta potente para fomentar el aprendizaje participativo y desarrollar cultura evaluadora. Así, la transparencia sobre los contenidos y conclusiones de la evaluación se convierte en un instrumento muy útil para la mejora de la gobernanza, lo que al final puede redundar en un aumento de la eficacia de los programas, al estimular la participación y la motivación de los diferentes grupos de stakeholders, facilitando el aprendizaje y la capacidad de comprensión de las relaciones causa-efecto subyacentes sobre los diferentes procesos.

En relación a la influencia del contexto aplicado a nuestro estudio de caso, si bien somos conscientes de la existencia de escenarios más favorables que otros a la hora de poner en práctica experiencias participativas, se pudo constatar cómo la adopción de 
estos modelos es eficaz incluso cuando la estructura organizacional en la que se enmarca el trabajo no resulta a priori la más adecuada. En este estudio de caso, y a lo largo de todo el proceso, nos fuimos encontrando con personas que, aunque en un principio eran reacias a invertir tiempo y esfuerzo en la evaluación, fueron modificando posteriormente su actitud una vez que tomaban conciencia sobre utilidad del proceso en el que estaban tomado parte. Este aspecto subraya la habilidad de la evaluación participativa para convertirse en una herramienta que pueda ayudar a flexibilizar y disminuir la organización jerárquica de las instituciones, haciéndolas más democráticas y comunicativas.

Por otro lado, en un escenario como el descrito, la existencia de un elevado número de clientes y usuarios potenciales a los que la evaluación debía satisfacer, podía crear tensiones que afectarían, de manera directa, al uso de la evaluación. En este sentido, el análisis realizado sobre el uso de los resultados y el proceso de la evaluación puso de manifiesto la importancia que la evaluación participativa alcanzó tanto para los participantes en el proceso como para audiencias más amplias. El hecho de que el informe se convirtiera en un documento de referencia para desarrollar un debate mucho más amplio sobre el futuro de la política de desarrollo rural en la región y su gobernanza fue, sin duda, un logro del modelo de evaluación utilizado.

Relativo a este último aspecto, creemos que, como equipo de evaluación, deberíamos haber abordado desde el inicio del proceso un análisis más fino y preciso de la estructura organizativa en la que nuestro trabajo se desenvolvía y haber llegado a ser capaces de buscar medios y/o vínculos para reforzar las relaciones entre la evaluación y su proceso participación con el nivel donde se toman las decisiones políticas, de forma que la gobernanza se convirtiera en el motor transformador de la intervención pública. Éste será, sin duda, un reto clave para el futuro.

\section{REFERENCIAS BIBLIOGRÁFICAS Y DOCUMENTALES}

Alkin, M. C., Christie, C. A. y Rose, M. (2006), “Communicating evaluation”, en I.F. Shaw, J.C. Green y M.M. Melvin, eds., Handbook of evaluation. Policies, programs and practices. Londres: SAGE.

Bachler, J. y Michie, R. (1995), “A new era in EU Regional policy evaluation? The apraisal of the Structural Funds", Regional Studies, 29: 745-751.

Burke, B. (1998), "Evaluating for a change: reflections on participatory methodology", en E. Whitmore, ed., Understanding and Practicing Participatory Evaluation. San Francisco: Jossey-Bass.

Centre for European Evaluation Expertise (1996), MEANS Handbook Collection (Methods for Evaluating Structural Policies). Luxemburgo: European Commission.

Chelimsky, E. (1997), "The coming transformation in Evaluation", en E. Chelimsky y W. R.Shadish, eds., Evaluation for the 21st Century. A Handbook. Londres: SAGE.

Chelimsky, E. (2006), "The purposes of evaluation in a democratic society", en I.F. Shaw, J.C. Green, y M.M. Melvin, eds., Handbook of evaluation. Policies, programs 156 and practices. Londres: SAGE. 
Connell, J.P, Kubisch, A. C., Schorr, L. B. y Weiss, C. H. (1995), New Approaches to Evaluation Community Initiatives: Concepts, Methods and Context. Washington: Aspen Institute.

Cousins, J. B. y Whitmore, E. (1998), “Framing participatory evaluation”, en E. Whitmore, ed., Understanding and Practicing Participatory Evaluation. San Francisco: Jossey-Bass.

Cousins, J. B. y Shulba, L. M. (2006), "A comparative analysis of evaluation utilization and its cognate fields of inquiry: current issues and trends", en I.F. Shaw, J.C. Green, y M.M. Melvin, eds., Handbook of evaluation. Policies, programs and practices. Londres: SAGE.

Díez, M. A. e Izquierdo, B. (2006), “La evaluación de los Fondos Estructurales de la Unión Europea”, Ekonomiaz, 60: 178-209.

Díez, M. A. (2001), "The evaluation of regional innovation and cluster policies: towards a participatory approach", European Planning Studies, 9(7): 907-923.

Díez, M. A. (2002), "Evaluating new regional policies: reviewing the theory and practice", Evaluation, 8: 285-305.

European Commission (2001), European Governance. A White Paper. Bruselas: Commission of the European Comunities, COM: 428-463.

European Policy Evaluation Consortium (2005), Study on the use of evaluation results in the Commission. Bruselas: European Commisssion. En línea: <http://ec.europa. eu/dgs/secretariat_general/evaluation/docs/eval_results_comm_2005_en.pdf.> (Consulta: 5 mayo 2008).

Feinstein, O. N. (2002), "Use of evaluations and the evaluation of their use", Evaluation, 8: 433-439.

Fetterman, D. M. (2001), Foundations of empowerment evaluation. Londres: SAGE.

Finne, H., Levin, M. y Nilssen, T. (1995), "Trailing research. A model for useful program evaluation", Evaluation 1: 11-31.

Forss, K., Rebien, C. y Carlsson, J. (2002), "Process use of evaluation. Types of use that precede lessons learned and feedback", Evaluation, 8: 29-45.

Green, J. (2006), "Evaluation, democracy and social change”, en I.F. Shaw, J.C. Green, y M.M. Melvin, eds., Handbook of evaluation. Policies, programs and practices. Londres: SAGE.

Green, J. (1998), "Stakeholder participation and utilization in program evaluation", Evaluation, 12: 25-35.

Gregory, A. (2000), “Problematizing participation. A critical review of approaches to participation in evaluation theory", Evaluation, 6: 179-199.

Guba, E. G. \& Lincoln, Y. S. (1989), Fourth Generation Evaluation. Newbury Park: Sage.

Kuhlmann, S. (1998), "Moderation of policy making? Science and Technology Policy evaluation beyond impact measurement-The case of Germany", Evaluation, 4: 130-148.

Monnier, E. (1995), Evaluación de la acción de los poderes públicos. Madrid: Instituto de Estudios Fiscales.

Murray, R. (2002), "Citizen's control of evaluations. Formulating and assessing alternatives", Evaluation, 8: 81-100.

Narayan-Parker, D. (1993), "Participatory evaluation tools for managing change in water and sanitation”, World Bank Technical Paper 207. Washington DC. 
Patton, M. Q. (1998), “Discovering process use”, Evaluation, 4: 225-233.

Patton, M. Q. (2001), "Evaluation, knowledge management, best practices and high quality lessons learned”, American Journal of Evaluation, 22: 329-336.

Patton, M. Q. (2008), Utilization-Focused Evaluation: The new century text. Londres: SAGE.

Plottu, B. y Plottu, E. (2009), “Approaches to participation in evaluation. Some conditions for implementation", Evaluation, 15: 345-359.

Ray, C. (2000), "Endogenous socio- economic development in the European Unionissues of evaluation", Journal of Rural Studies, 16: 2-14.

Rist, R. y Stame, N., eds., (2006), From studies to streams: managing evaluation systems. New Brunswick: Transactions Publishers.

Stame, N. (1999), "Small and Medium Enterprise Aid Programs: intangible effects and evaluation practice", Evaluation and Program Planning, 22: 105-111.

Stame, N. (2006a), "Governance, democracy and evaluation", Evaluation, 12: 7-16.

Stame, N. (2006b), "Complex policies and evaluative streams of knowledge”, en R. Rist y N. Stame, eds., From studies to streams: managing evaluation systems. New Brunswick: Transactions Publishers.

Suarez-Herrera, J. C., Springett, J. y Kagan, C. (2009), “Critical conections between participatory evaluation, organizational learning and intentional change in pluralistic organizations", Evaluation, 15: 321-342.

Subirats, J. (2006), “Catorce puntos esenciales sobre evaluación de políticas públicas con especial referencia al caso de políticas sociales”, Ekonomiaz, 60: 18-37.

Themessl-Hubber, M. T. y Grutsch, M. A. (2003), "The shifting locus of control in participatory evaluations", Evaluation, 9: 92-111.

Torres, R., Preskill, H. y Piontek, M. (2005), Evaluation strategies for communicating and reporting. Londres: SAGE.

Valovirta, V. (2002), "Evaluation utilization as argumentation”, Evaluation, 8: 60-80.

Viñas, V. (2009), “The European Union's drive towards Public Policy Evaluation: the case of Spain”, Evaluation, 15: 459-472.

Weiss, C. H. (1987), "Evaluating social programs: what have we learned?", Society, 25 (1): 40-45.

Weiss, C. H. (1998), "Have we learned anything new about the use of evaluation?", American Journal of Evaluation, 19(1): 21-33.

Weiss, C. H. y Bucuvalas, M. J. (1980), “Truth tests and utility tests: decisión makers' frames of reference for social science research", American Sociological Review, 45: 302-313.

Whitmore, E., ed., (1998), "Understanding and Practicing Participatory Evaluation”, New Directions for Evaluation, 80. 


\section{ANEXO}

\section{Información suministrada}

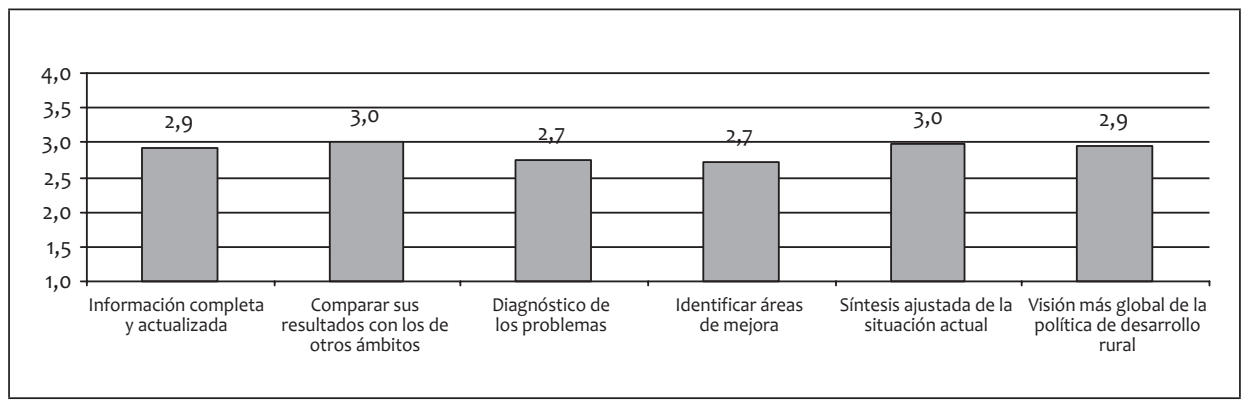

Fuente: elaboración propia.

\section{Mejora de la aplicación}

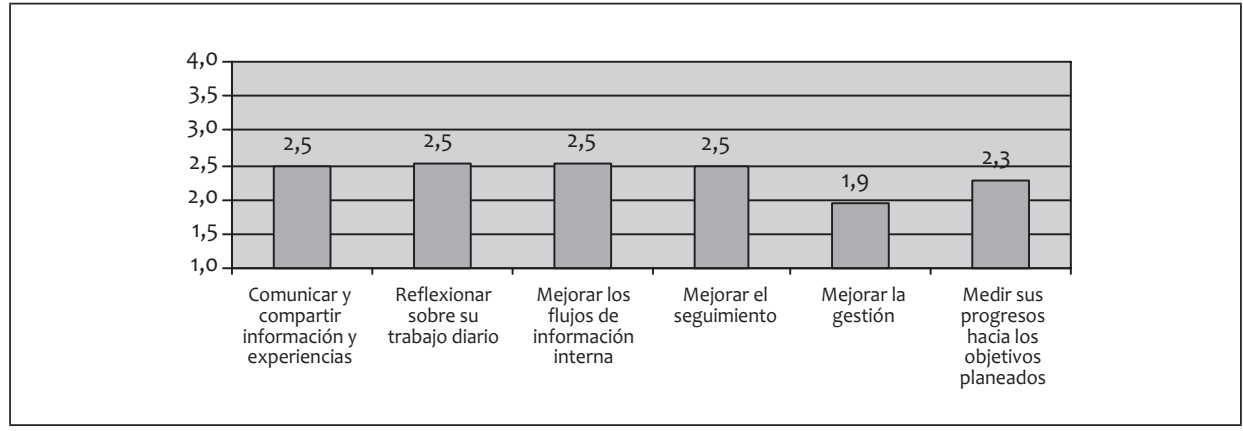

Fuente: elaboración propia.

\section{Conocimientos adquiridos}

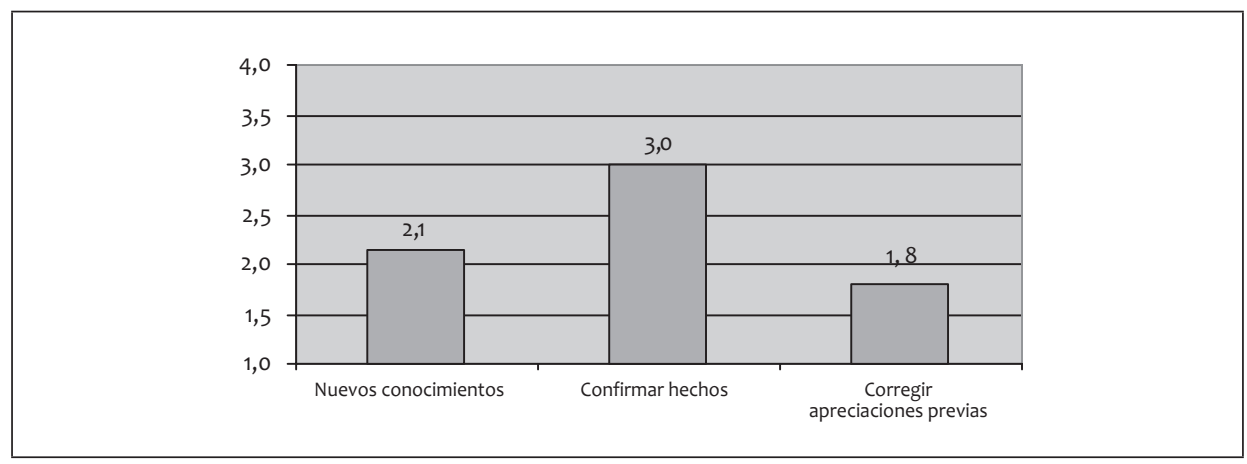

Fuente: elaboración propia. 


\section{Impacto potencial sobre la política}

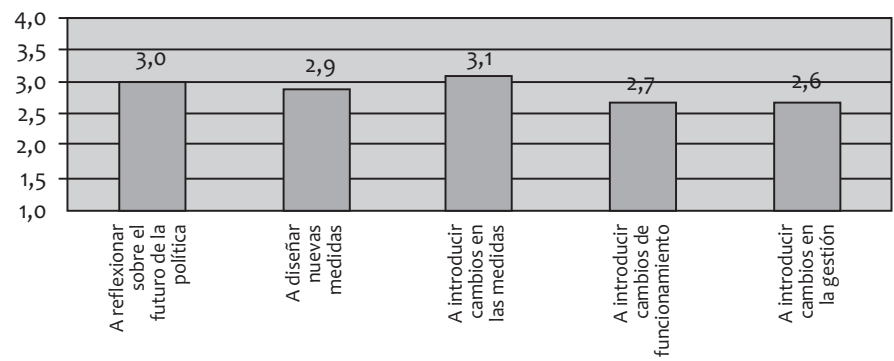

Fuente: elaboración propia.

\section{Utilidad global}

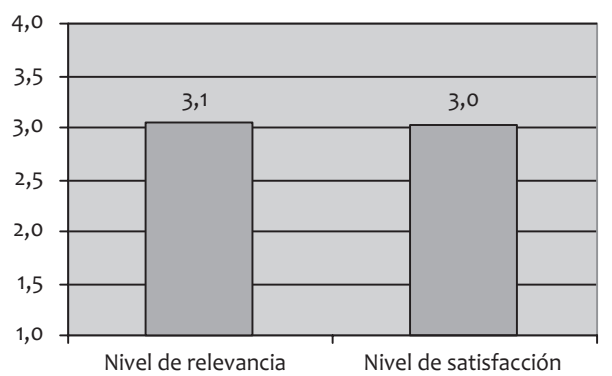

Fuente: elaboración propia.

\section{Metodología}

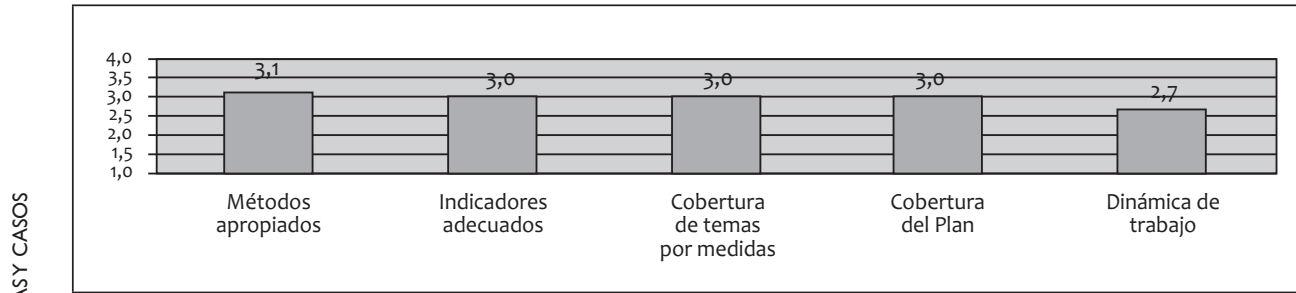

Fuente: elaboración propia.

Recibido: 26 de febrero de 2010

Aceptado: 25 de mayo de 2010 